\title{
Effects of mechanical deformation: Exoemission
}

\author{
G MUKHOPADHYAY
}

Department of Physics, Indian Institute of Technology, Bombay 400076, India

\begin{abstract}
An introduction to some of the physical effects (e.g. exoemission, acoustic emission and mechanoluminescence) associated with the mechanical deformation of solids is presented. Greater emphasis has been given to exoelectron emissions. Experimental information and plausible mechanisms for exoemission have been described briefly. In particular, exoelectron emission from metals and oxide-coated metals has been discussed at some length, with the hope of generating a common interest among physicists and metallurgists.
\end{abstract}

Keywords. Mechanical deformation; stress; defects; dislocations; electron emission.

\section{Introduction}

There exist a number of effects associated with the mechanical deformation of solids. One class of effects is the emission of particles (charged and/or neutral), light and sound, when a material is undergoing deformation or is subjected to a constant strain rate. The emission of particles (in particular, electrons) is called exoelectron emission. The emission of light usually occurs in the form of luminescence from those materials which contain luminescent sources, and such an effect when induced by mechanical deformation is called mechanoluminescence. The emission of sound or stress waves is known to occur from a variety of materials, and is usually called acoustic emission. We shall describe here these effects and their understanding at the present time, with particular emphasis on exoemission, especially from metals and metal oxides during mechanical deformation. We shall see that these effects have some common features, require a great deal of future study and promise to be excellent tools for applied research (especially for metallurgists) in the field of nondestructive testing.

\section{Exoemission}

The phenomenon of exoemission (EE) was reported quite a long time ago (McLennan 1902). However, it was mainly after the introduction of the Geiger tube in the 1920's that the effect was truly recognised. Freshly-prepared Geiger tubes gave high and irregular counting rates for the first few hours or days, before functioning normally, and this led to the discovery that freshly machined metal surfaces emit electrons. This phenomenon was first carefully investigated by Kramer (1950) and sometimes referred to as the Kramer effect. Kramer himself found that all freshly prepared metal surfaces gave off electrons and suggested that the electrons gained the required energy for coming out of a metal by acquiring some of the energy released in the exothermal processes occurring on the surface. The prefix 'exo-' originates from this explanation. Although our present understanding indicates that the actual explanation of the 
phenomenon is not as simple as this, the name has survived. It now appears that EE occurs for almost all materials in varying degree.

\subsection{Main features of $E E$}

The main features of EE occurring in various materials can be summarized as follows:

(i) The material exhibiting EE must be 'excited' externally to produce a perturbation in the form of a structural change. The external excitation may be in the form of cold working, irradiation by electromagnetic radiation (Uv or higher frequencies), particle bombardment ( $\alpha, \beta$-rays, neutrons, protons, slow electrons, ions), exposure to gases, abrasion, mechanical deformation, etc.

(ii) The effect is strongly correlated with relaxation processes in the material, both causally and temporally, and it appears only after the generation of the perturbation. The observed time dependence can often be fitted by one or more decaying exponentials. The corresponding time constants refiect the presence of one or more relaxation processes, which normally depend strongly on the amount and nature of the defects in the system. It is this aspect of the EE process that makes it an attractive nondestructive technique of characterizing a material for its defect contents.

(iii) Unlike stationary effects, such as photoemission (PE) and thermionic emission (TE), EE is a nonstationary process, i.e. the EE flux is of transient character. Further, the effect is rather weak compared to PE or TE, with a EE flux typically in the range of $10^{-13} \mathrm{~A} / \mathrm{m}^{2}$.

(iv) The emission may be spontaneous or may require external stimulation, in addition to the external excitation. The stimulation may be in the form of increased temperature (thermally-stimulated exoemission (TSEE)) or electro-magnetic radiation (photo-stimulated exoemission (PSEE)), usually in the optical range. The EE observed without any photostimulation is usually referred to as 'dark emission'. Normally TSEE is observed non-isothermally, although it can also be observed isothermally. For PSEE, it is necessary that the photon energy is less than the work function of the material under study. Otherwise normal PE will accompany EE making data analysis rather difficult. PSEE can enhance a normal 'dark EE' intensity by a factor as large as $10^{4}$ or more. Both TSEE and PSEE have been correlated with several other effects, such as thermally stimulated luminescence (TSL), electron-stimulated desorption (ESD), thermally stimulated conductivity (TSC), thermally stimulated depolarization currents (TSDC), and thermally stimulated desorption (TSD), depending on the material studied. Observations of these effects enhance the understanding of the EE process and are therefore generally desirable. There does not appear to have been any attempt as yet to correlate EE with acoustic emission or mechanoluminescence.

(v) The EE rate is strongly correlated with the work function $\phi$ of the system. This correlation is of major relevance for PSEE as well as TSEE, and it is necessary to measure the work function simultaneously by an independent method during an EE observation, to facilitate understanding of the EF process.

(vi) The EE effect has two components, one of which is volume dependent and the other, surface dependent. For a particular system one or both may occur depending on the material and its surface condition as well as its surrounding gas medium. The surface effect is strongly related to the surface condition, and in a controlled EE experiment it is often desirable to have an ultra-high vacuum $\left(\sim 10^{-10}\right.$ Torr) to separate the surface effect from the volume effect. It is also desirable to have an experimental arrangement for characterizing the surface condition of the sample. 
(vii) EE emission can occur continuously, or in large bursts, or with the latter superimposed over the former. It is usually believed that the continuous emission is related to the motion of defects in the sample, whereas the emission in bursts is related to the propagation of cracks (or micro-cracks). Thus the nature of the EE may reveal the nature of its source.

\subsection{Experimental arrangement}

The apparatus for measuring EE consists of an excitation unit, a stimulation unit and a detection unit, at tached to an ultrahigh vacuum (UHv) chamber containing the sample. It should be equipped with an Auger spectrometer and a quadrupole mass analyzer for structural and chemical analysis of the surface of the sample and for the identification of the chemical composition of the residual gases in the chamber. A facility for measuring the work function (eg., by the Kelvin or Fowler method) is desirable. In addition, a LFED facility may be used for characterization of the surface structure. A quartz window on the chamber will facilitate optical measurements (e.g. luminescence, optical absorption, etc.), which often prove valuable for understanding EE.

An apparatus and the technique for observing TSEE has been described by Glaefeke (1979). We describe here an apparatus for observing EE or PSEE (figure 1). This is perhaps one of the most sophisticated versions of this type, developed and used by Rosenblum et al (1977) for studying EE during the mechanical deformation of a dogbone shaped sample (marked $\mathrm{S}$ in figure 1) of $\mathrm{Al}, \mathrm{Ni}$ or $\mathrm{Ti}$, with and without oxidised layers. The details of their experimental arrangement are described briefly here. The excitation unit consists of an arrangement for producing uniaxial tensile strain at a constant rate. The sample placed inside an UHV chamber (part of a LEED apparatus) is clamped at both ends, with the fixed end attached to a load cell (LC) and the other end attached to a pulling rod coupled to a linear variable differential transformer (LVDT) for measuring the displacement and thereby the strain rate. Strain at a constant rate is applied at intervals, causing EE in bursts. The vacuum chamber (UHVC) is equipped with a ion-gun (IG) for in-situ cleaning of the sample surface. No stimulation unit was used by Rosenblum et al (1977), although a LiF window (W) is provided for the purpose of photostimulation. In such experiments the EE flux is usually weak $\left(10^{4}-10^{6}\right.$ particles $/ \mathrm{sec} / \mathrm{m}^{2}$ ) and it is desirable to have a single-event charged particle and photon
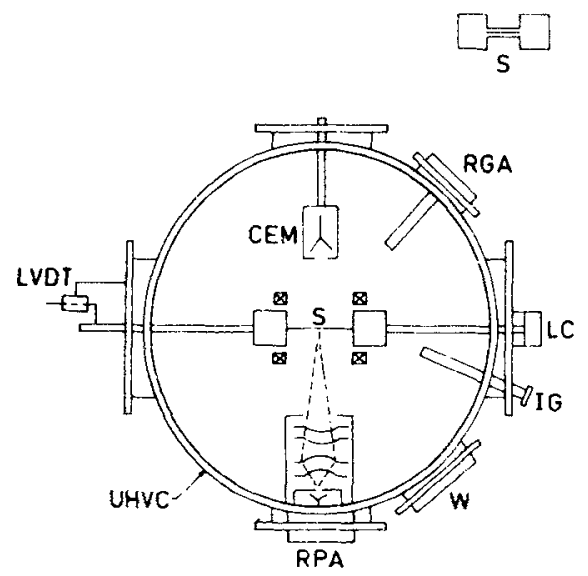

Figure 1. Schematic diagram for EE apparatus. (Rosenblum et al 1977). 
detector as part of the detection unit. Rosenblum et al used a channel electron multiplier (CEM in figure 1) with an input cone (for focussing and accelerating the emitted particles into CEM) with an appropriate shielding and magnetic-discrimination scheme (for identifying the charged particles as electrons, or negative or positive ions). The shield ensured that the electrostatic field developed between the emitting surface and the cone was due to the CEM's biasing potential relative to the specimen rather than to the potential across the CEM. A retarding potential analyser (RPA) was used for Auger and photoelectron spectrometry for studying and characterizing the surface condition of the sample. For the detection of photons (presumably arising from the mechanoluminescence effect), a miniature single-photon counter tube (Bendix BX 754) was used in place of the (removable) RPA unit. For measuring the energy distribution, an electron spectrometer of high resolution (retarding grid potential analyser-RGA in figure 1) was specially designed to suit the way the strain was applied and the EE observed. However, the energy in the energy distribution could be measured only with respect to the Fermi level of the retarding grid, since the work functions of the oxides were uncertain, especially under mechanical strain. Figures 2-5 show the results of EE from Al with an oxide $\left(\mathrm{Al}_{2} \mathrm{O}_{3}\right)$ layer, observed by Rosenblum et al $(1977,1977 \mathrm{a})$. In figure 2, curves 1-3 correspond to electron emission intensity plotted against strain for samples with oxide thickness of $2000 \AA, 250 \AA$ and $50 \AA$ respectively; the oxides for $2000 \AA$ and $250 \AA$ were grown anodically while the $50 \AA$ thick oxide was grown naturally (i.e., natural airformed oxide). Curve- 4 corresponds to negative ion emission from the $2000 \mathrm{~A}$ thick oxide sample. Curve 5 is the stress-strain curve for the sample. In all cases a constant strain rate of $\dot{\varepsilon}=2.2 \times 10^{-4} \mathrm{sec}^{-1}$ was used at an ambient pressure of $10^{-10}$ Torr. In figure 3 , curve 1 corresponds to photon emission from the $2000 \AA$ thick oxide sample

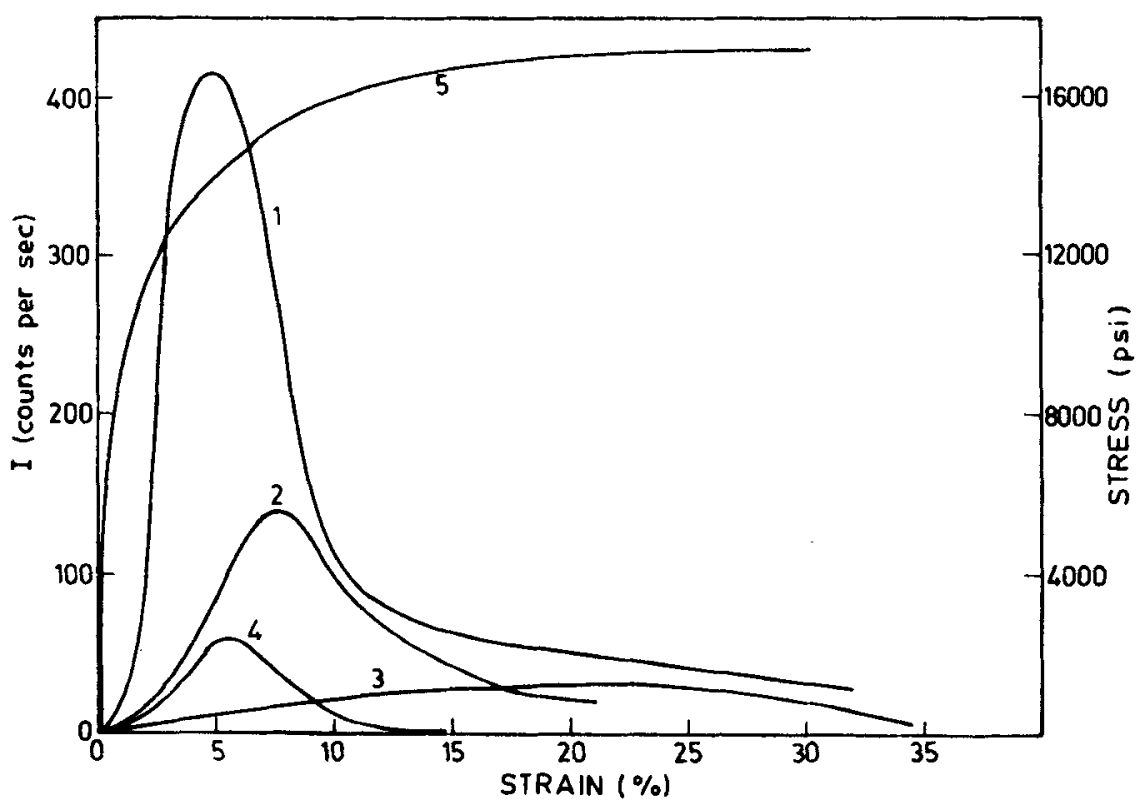

Figure 2. Electron emission intensity vs strain: 1. $d$ (oxide thickness) $=2000 \mathrm{~A}$; 2. $d=250 \mathrm{~A}$; 3. $d=50 \mathrm{~A}$; Negative ion emission: 4. $d=2000 \mathrm{~A}$; stress-strain relation: 5. Strain rate $\dot{\varepsilon}=2.2 \times 10^{-4} \mathrm{sec}^{-1}$ (Rosenblum et al 1977, 1977a). 


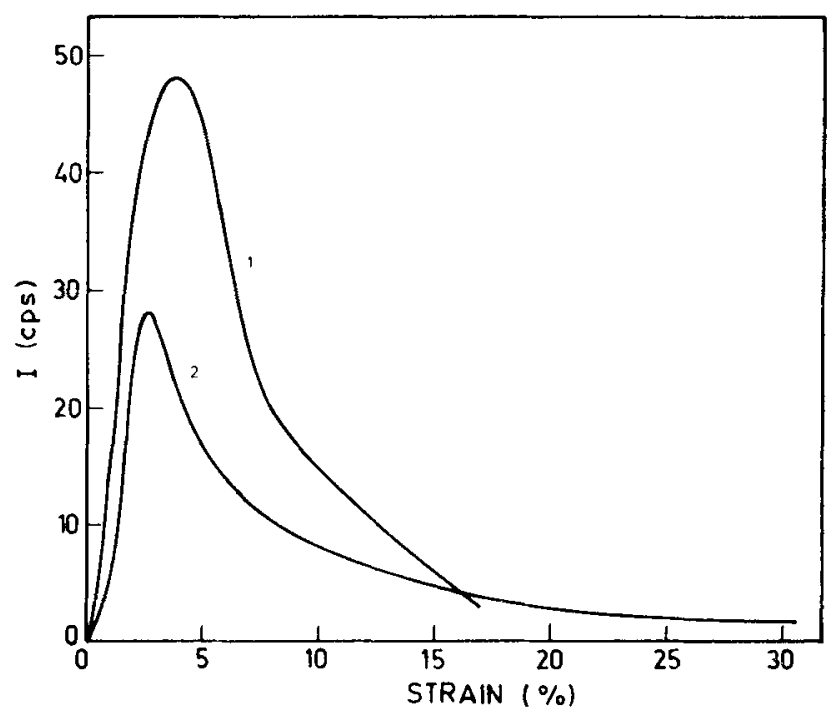

Figure 3. Photon emission intensity $v$ s strain: $1 . \dot{\varepsilon}=2.2 \times 10^{-4} \mathrm{sec}^{-1}$. Positive ion emission: 2. $\dot{\varepsilon}=5.3 \times 10^{-4} \sec ^{-1} d=2000$ A (Rosenblum et al 1977, 1977a).

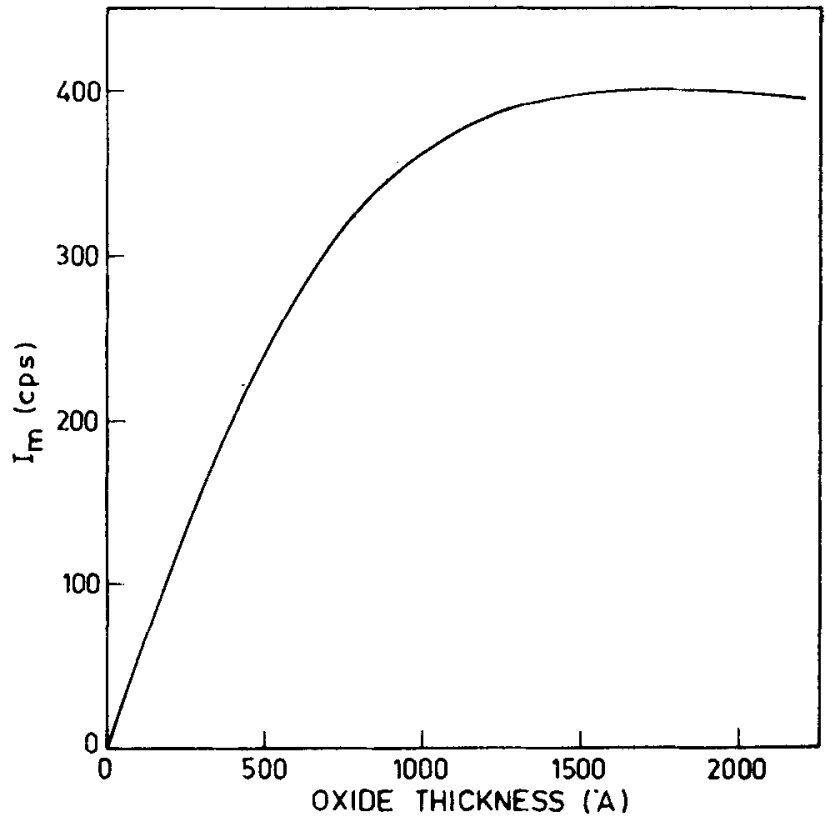

Figure 4. Maximum electron emission intensity $v$ s oxide thickness: $\dot{\varepsilon}=2.2 \times 10^{-4} \mathrm{sec}^{-1}$ (Rosenblum 1977a).

for the same strain rate as above, while curve 2 corresponds to positively charged ion emission for the same sample at a strain rate of $5.3 \times 10^{-4} \mathrm{sec}^{-1}$. The general features of the intensity as a function of strain are the same for the emission of electrons, positively or negatively charged ions, or photons. Figure 4 shows the peak electron 


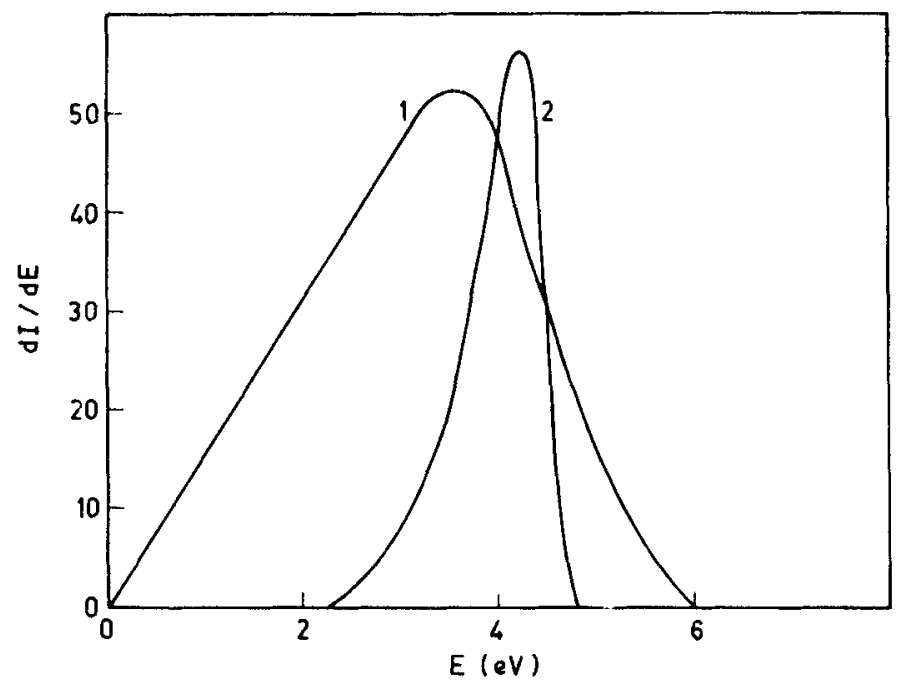

Figure 5. Electron emission intensity per unit energy interval vs energy: 1. EE; 2. TE (Rosenblum et al 1977, 1977a).

emission intensity as a function of the (anodically grown) oxide thickness for $\dot{\varepsilon}=2 \cdot 2$ $\times 10^{-4} \mathrm{sec}^{-1}$ at $10^{-10}$ Torr. In figure 5, curve 1 shows the electron emission intensity per unit energy interval as a function of energy, for $\dot{\varepsilon}=2.2 \times 10^{-4} \mathrm{sec}^{-1}$, while curve 2 is the corresponding result for thermionic emission, both for the $2000 \AA$ thick oxide sample. These results provide an idea of the characteristics of EE from oxide-coated metals.

Another interesting experiment is due to Baxter (1973), who studied exoelectron emission by photostimulation during fatigue cycling of aluminium. The emission intensity was measured as a function of the stress cycle number. While Baxter's experimental arrangement was different (see Baxter 1973 for details), the apparatus of figure 1 can be employed in principle, with additional arrangements for stress cycling. A dogbone-shaped sample (as in figure 1) was clamped rigidly at one end and other end was driven by an external cam, producing fatigue cycling in the bending mode. Photostimulation was done by a small spot of light (15 to $70 \mu \mathrm{m}$ in diameter, according to the sensitivity), scanning the sample lengthwise (for example, along the line on the narrow strip of the sample $S$ in figure 1). The location of the light spot on the sample was monitored by an optical arrangement, and the intensity of PSEE from different locations determined accurately. The emission was localised at the region of high local strain. With increasing cycling, an initial localised peak (or peaks in case of more than one detectable region of high local strain) grew and fresh peaks also emerged. The specimen ultimately failed locally in the vicinity of this primary peak. For the Al sample, at high strain (i.e., $\pm 4.6 \times 10^{-3}$ ) emission could be detected after only $0.07 \%$ of the fatigue life of 14000 cycles (i.e., 10 cycles), whereas at lower strain $\left( \pm 2 \times 10^{-3}\right.$ ), after $0.7 \%$ of the fatigue life of 140000 cycles. The results for steel were also similar in nature (except at high cycling), with emission detectable after only $1 \%$ of the fatigue life. The stability of the localized emission was studied by interrupting fatigue cycling at various stages and monitoring the emission peak for an hour before continuing with the 


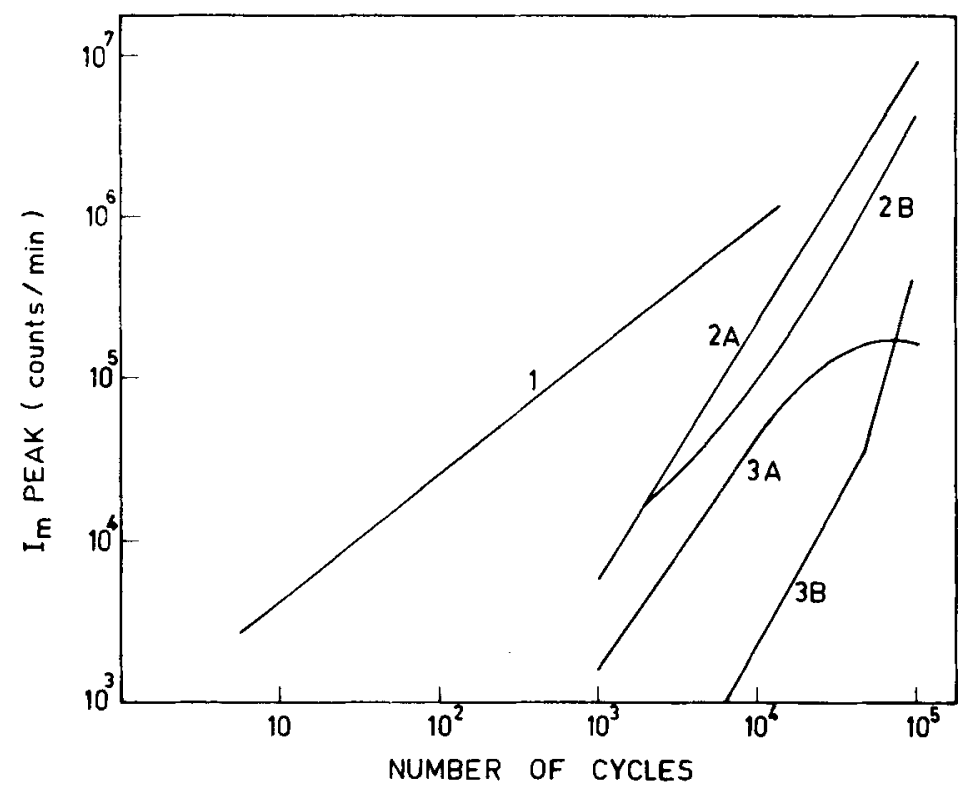

Figure 6. Maximum electron emission intensity vs number of fatigue cycles: 1. Al (fatigue strain $\pm 4.6 \times 10^{-3}$ ): $2 \mathrm{~A}, \mathrm{~B} \mathrm{Al}$ (fatigue strain $\pm 2 \times 10^{-3}$ ): $3 \mathrm{~A}, \mathrm{~B}$. steel (fatigue strain $\pm 2.5 \times 10^{-3}$ ) (Baxter 1973). (A and B correspond to different locations in the sample).

cycling. The emission was extremely stable (intensity independent of time), except for a slight transient during the first few minutes following the interruption in fatigue cycling. Figure 6 summarises the results reported by Baxter. Curve 1 corresponds to a primary peak (localised on the sample) emission intensity for Al fatigued at a strain of $\pm 4.6 \times 10^{-3}$ (fatigue life 14000 cycles). Curves $2 \mathrm{~A}$ and $2 \mathrm{~B}$ correspond to emission of almost the same initial intensity from two different locations on the $\mathrm{Al}$ sample fatigued at a strain of $\pm 2 \times 10^{-3}$ (fatigue life 140000 cycles); the sample eventually failed at the location corresponding to the curve $2 \mathrm{~A}$. Curves $3 \mathrm{~A}$ and $3 \mathrm{~B}$ correspond to steel fatigued at a strain of $\pm 2.5 \times 10^{-3}$ (fatigue life $1.06 \times 10^{5}$ cycles). Three dominant peaks (from three different locations) were traced, with two following $3 \mathrm{~A}$ and the third, $3 \mathrm{~B}$. The latter (3B), initially small in magnitude, dominated after $10^{5}$ cycles, and the specimen eventually failed there. It was believed that the growth of localised emission with fatigue cycling was controlled by crack formation and propagation. Curves 1 and 2 in figure 6 were thought to represent simple uncomplicated cases of crack propagation. The change-over behaviour occurring in the steel sample (represented by curves $3 \mathrm{~A}$ and $3 \mathrm{~B}$ ) was regarded as a situation in which the initial major crack encountered an obstacle preventing its propagation, further deformation being accommodated by the rapid growth of another crack. The role of the diffusion of point defects and surface conditions were thought to be of no importance in view of the stability of the emission. In any case, it seems obvious from the above mentioned results that the intensity of localized electron emission may be regarded as a measure of the extent of the localized fatigue damage. It is this particular aspect that makes this type of experiment very attractive as a nondestructive testing device. 


\subsection{Mechanism and general understanding}

The mechanisms for EE have always been a subject of controversy, partly because each worker or group of workers has tried to explain the experimental results through phenomenology suitable for that particular experimental situation (which is not easily reproduced), and mainly because of the very complex nature of the EE phenomenon. We now describe briefly some of the mechanisms based on observations under specific situations (see Glaefeke 1979 for further details).

2.3a Excitations induced chemically: Spontaneous EE can occur when chemically reactive gases interact with initially clean metal surfaces (Bohun 1961; Bohun et al 1965; Delchar 1967; McCarrol 1969; Gesell et al 1970; Brus and Comas 1971; Born and Linke 1976). The process involved here is adsorption, the subsequent emission occurring from the surface. An adsorption process can be either physical (physisorption) or chemical (chemisorption) in nature. In physisorption, the adsorption-desorption equilibrium (at constant $\mathbf{T}$ ) is reached without activation. The heat of physisorption is typically of the order of $0.1 \mathrm{eV}$ per species, which is too small to trigger EE. In contrast, chemisorption requires an activation energy and is preceded by physisorption. The heat of chemisorption is of the order of several $\mathrm{eV}$ per species, sufficient for EE. Compared to physisorption which is reversible and forms multilayers, chemisorption is partially irreversible and ceases at monolayer coverage unless the adsorbed species diffuses into the bulk of the substrate. Such diffusion does occur in the case of oxidation and growth of the oxide layer. The rate of chemisorption may be determined from the chemical kinetic rate equation when the chemical reaction velocity is rate-determining, or from Fick's law of diffusion when the diffusion process is rate-limiting. The EE rate is then assumed to be proportional to the rate of chemisorption. Analysis along these lines then indicates a maximum in the EE current as a function of time. When more than one species is chemisorbed, one would expect a maximum corresponding to each species. Thus, the two observed maxima in the EE from clean $\mathrm{Mg}$ surfaces exposed (by abrasion) to $\mathrm{O}_{2}$ and $\mathrm{H}_{2} \mathrm{O}$ in the dark have been attributed to the two different adsorbing species (Gesell et al 1970). It has also been found from a simultaneous measurement of the work function that the maximum in the EE corresponds to a minimum in the work function $\phi$. Thus, the EE has been attributed to (a) the lowering of the work function due to adsorption with a dipole moment of the adsorbed complex favouring emission, and (b) transfer of the released heat of chemisorption to generate surface plasmons which in turn transfer their energy to electrons causing EE. For Al, a substantial decrease in the work function $\phi$ (with the minimum in $\phi$ coinciding with the maximum in dark EE) during adsorption of water has been reported (Born and Linke 1976, from $4.5 \mathrm{eV}$ to $1.3 \mathrm{eV}$; Wortmann 1978 ).

Spontaneous EE can also occur during thermally stimulated desorption (TSD) without any preliminary excitation, and such TSEE is sometimes referred to as self-excited EE (Bohun 1961; Krylova 1976; Krylova et al 1976). For e.g., highly hydrated oxides $\left(\mathrm{Al}_{2} \mathrm{O}_{3}\right.$, $\mathrm{MgO}$ etc.) and alkali halides ( $\mathrm{NaCl}$ etc.) show a strong gas desorption accompanied by EE (Krylova 1976). It is believed that the active species at the surface recombine with the sites which have become vacant through desorption due to thermal stimulation. The released recombination energy is then responsible for this TSEE (Krylova et al 1976).

TSEE has been observed during heterogeneous catalytic reaction, for example during catalytic oxidation of hydrogen and carbon monoxide on $\mathrm{NiO}, \mathrm{ZnO}$ and platinum 
black (Krylova 1976). Since both TSEE and catalysis depend on chemical thermodynamics, a relationship between the two is to be expected. Indeed, a reproducible linear relation between the two has been demonstrated for catalytic oxidation (Krylova 1976).

2.3b Excitation by $U V$ and $x$-rays: Some alkali halides (especially those with small electron affinity at the surface, e.g., chlorides and fluorides), during exposure to uv light, exhibit PSEE when photostimulated with an additional uv light source. TSEE may be observed during thermal annealing. The mechanism for $\mathrm{EE}$ is believed to be a two-step photothermal process involving colour centres generated by the exciting uv light. The first step is the optical transition from the colour centre to the conduction band, and the second step is the thermionic emission from the conduction band to vacuum. The stimulation spectra (i.e., PSEE as a function of the stimulating wavelength) have been studied extensively and found to give information about the colour centres similar to that yielded by their optical absorption spectra (Ford et al 1970; Bichevin et al 1971; Nink and Holzapfel 1973; Maiste et al 1973; Kortov et al 1982; Tale et al 1982).

TSEE has been reported after excitation with $\mathrm{x}$-rays, and a correlation between TSL and TSEE established (Holzapfel and Krystek 1976). TSEE from oxides irradiated with $\mathrm{X}$-rays has also been investigated (Rudolf and Glaefeke 1982). In most cases, a direct liberation of electrons from traps (produced by irradiation) is assumed to explain the EE.

2.3c Excitation by electrons: EE has been observed from materials during exposure to electron beams. However, this occurs only for insulators. So far, atomically clean metal surfaces have not exhibited EE on irradiation, and the presence of oxide or adsorbed species seems to be absolutely essential for EE observation here. This seems to be true also for semiconductors. The EE behaviour is strongly dependent on the energy of the exciting electron beam. For low energy electrons $(0.2$ to $30 \mathrm{eV})$ the adsorbed species on the surface and the surface states are affected and here EE is essentially a surface phenomenon. These EE spectra are similar to the UV-excited spectra, and electronstimulated desorption (ESD) can occur. ESD can influence TSEE spectra appreciably. Concepts involving chemical reactions at the surface are utilized to correlate the two (Jakowski and Glaefeke 1976; Euler and Scharmann 1976; Brunsmann and Scharmann 1977; Kirihata and Akutsu 1979). For moderate energy electrons (several hundred to several thousand $\mathrm{eV}$ ), volume states are excited, generating a complicated space charge inside the insulator. This in turn produces a positive-negative double layer whose electric field (as large as $10^{6} \mathrm{~V} \mathrm{~cm}^{-1}$ ) causes EE (Fitting et al 1979; Fitting 1981). Systems studied, among others, are $\mathrm{Al}_{2} \mathrm{O}_{3}$ (Krylova 1971; Schlenk 1976; Chrysson and Holzapfel 1980). ZnO (Krylova 1971; Hiernaut et al 1972; Kriegseis and Scharmann 1975), $\mathrm{NiO}$ (Krylova 1971; Hiernaut et al 1972), $\mathrm{SiO}_{2}$ (Krylova 1971; Jakowski and Glaefeke 1976), BeO (Euler and Scharmann 1976; Scharmann and Wiessler 1980) and $\mathrm{NaF}$ (Brunsmann and Scharmann 1977).

2.3d Excitation by nuclear radiation: Reproducible EE has been observed from a number of substances using nuclear radiation as the source of excitation. Among the substances studied are alkali halides, alkaline-earth halides, sulphates, sulphides and a number of metal oxides. TSEE from $\mathrm{BeO}$ and $\mathrm{LiF}$ have been studied extensively because of their dosimetric applications (see Glaefeke 1979 for details). Simultaneous measurement of TSL and TSEE from the same emitter can discriminate between those types of radiation which cause bulk effects (TSL) and those causing surface effects (TSEE). 
For example, both $\alpha$ - and soft $\beta$-irradiation yield high TSEE intensity (since the total energy is absorbed near the surface) but rather weak TSL, in $\mathrm{BeO}$. On the other hand, $\gamma$ irradiation causes very weak TSEE but a very strong TSL. Thus, TSEE and TSL from $\mathrm{BeO}$ can distinguish between $\alpha$ (or soft $\beta$ ) and $\gamma$ doses. Fast neutrons can cause EE via recoil protons or $\alpha$ radiation, and the corresponding TSEE may be separated from the $\gamma$-ray TSEE by a subtraction procedure to determine the fast neutron dosage. BeO dosimeters based on these properties may have a dose response range from as low as $10^{-6} \mathrm{rad}$ to $10^{2} \mathrm{rad}$. Compared to conventional dosimeters, however, a TSEE-based dosimeter has some technical shortcomings, such as a strong dependence of the TSEE on the mechanical treatment and the ambient atmosphere of the detecting material. This may be responsible for its lack of commercial usage.

2.3e Excitations through direct structural change: EE has been observed from a number of materials in which excitation is brought about by direct structural changes. Such changes can be induced by external application of stress, or by generating stress internally through heat treatment of the material.

EE has been observed from metals with atomically clean surfaces in ultrahigh vacuum. Kortov et al (1971) observed EE during plastic deformation of Al. Mints et al (1973) tried to correlate EE with the process of recovery and recrystallization of metals. Mechanical deformation generates a high concentration of defects (vacancies and dislocations). The relaxation of the stress is accompanied by the diffusion of vacancies towards the surface and the recovery of dislocations. These defects can rearrange themselves exothermally, and the localized release of thermal energy may be taken up by electrons. However, emission of these electrons would require a substantial lowering of the work function $\phi$, which is believed to be due to high roughness on a clean metal surface (Lewis 1954; Rhead 1977). Pardee and Buck (1977) and Buck et al (1977) considered the roughness-enhanced coupling of incident light with surface plasmons (which can transfer their energy to electrons) to explain PSEE.

EE from oxide covered metals has been observed both in the dark as well as with photostimulation during mechanical deformation. Since most metals in practical situations are oxidised at the surface, these EE observations are of considerable applied interest. The dark emission under UHV has been observed only during mechanical deformation or abrasion of oxide covered metals (Gieroszynski and Sujak 1965; Sujak et al 1965; Brotzen 1967; Sujak and Gieroszynski 1970; Linke 1970; Kortov et al 1970; Arnott and Ramsey 1971; Kortov and Myasnikov 1972; Kelly and Himmel 1976; Rosenblum et al 1977, 1977a). Photostimulated emission during abrasion or plastic deformation of oxide-coated metals can be very large as compared to the dark emission (by a factor of $10^{4}$ or more) and a number of oxidised metals have been investigated exploiting this fact (Grunberg 1958; Von Voss and Brotzen 1959; Pimbley and Francis 1961; Mueller and Pontinen 1964; Claytor and Brotzen 1965; Gieroszynski and Sujak 1965; Sujak et al 1965; Sujak and Gieroszynski 1970; Baxter 1973; Shorshorov et al 1976; Baxter and Rouze 1978; Wortmann 1978; Dickinson et al 1978; Komai 1978). Among various oxide coated materials (eg., $\mathrm{Al}, \mathrm{Ni}, \mathrm{Steel}, \mathrm{Ti}, \mathrm{Mo}$, etc.), the most widely studied one is $\mathrm{Al}_{2} \mathrm{O}_{3}$-coated Al. Qualitatively the same emission behaviour has been observed when the oxide covered metal is mechanically abraded or plastically deformed under tension. In the case of tensile strain, dark emission occurs only during a change in the strain and ceases abruptly when the deformation stops. Similarly, when photostimulated, considerable enhancement in electron emission occurs only during constant 
strain rate, the emission being regarded as burst emission, even though a continuous component (much smaller in magnitude) may be present. In case of burst emission, typically, for a constant strain rate, the emission increases to a maximum followed by a monotonic decrease with increasing deformation or strain (see figure 2). The emission depends on the strain rate as well as the oxide thickness (see figure 4). The burst emission has been associated with the propagation of cracks or micro-cracks. PSEE from thick oxide layers has been attributed to the propagation of cracks perpendicular to the stress direction, independently of the underlying micro-structure of the metal. In thin oxide layers, it has been attributed to fracture at slip steps (Baxter and Rouze 1978).

The continuous EE is believed to arise from two components, one controlled by the diffusion of point defects (generated by strain) towards the surface, and the other controlled by the relaxation of dislocations. One may dominate over the other, or both may be present. For example, Pimbley and Francis (1961) could explain EE from abraded oxidised Al through a model based on the diffusion of vacancies, while Claytor and Brotzen (1965) explained EE from mechanically deformed oxidised $\mathrm{Al}$ in terms of the diffusion of other point defects. On the other hand, Mueller and Pontinen (1964) studied EE from freshly abraded as well as from abraded and aged samples of oxidised $\mathrm{Al}$ and concluded against the defect diffusion mechanism. However, Shorshorov $e t$ al (1976) could correlate PSEE during plastic deformation with the generation of dislocations as well as vacancies by simultaneously studying the behaviour of these defects (using metallographic methods). They found no diffusion of vacancies but found relaxation of dislocations in molybdenum, while both phenomena occurred in $\alpha$ iron.

The burst emission, especially in dark, is supposed to originate from cracks or microcracks which develop in the oxide as soon as the local surface strain in the underlying metal substrate exceeds the ultimate tensile strain of the oxide layer (Sujak 1964). Originally it was believed that the opposing surfaces of a propagating fissure (microcrack) become charged, providing a strong electric field that causes field emission (Gieroszynski and Sujak 1965). This so-called electrified-fissure model for EE was reinvestigated by Arnott and Ramsey (1971) who restricted its applicability to polar substances. For non-polar substances, they proposed to interpret EE in terms of rearrangement of dangling bonds created during the propagation of cracks. Rabinowicz (1977) has elucidated this model, but also questioned its feasibility. Rosenblum et al (1977a) believe that the release of strain energy in the vicinity (e.g. the tip) of a propagating crack results in local heating to a temperature (estimated to be $\sim 3000 \mathrm{~K}$ for $\mathrm{Al}$ ) sufficient to cause thermionic emission from the walls. However, they also observed emission of positive and negative ions, which indicates that field-assisted emission of electrons cannot be ruled out.

EE during heat treatment of materials (without external excitation) arises from the internal mechanical stress generated thermally due to the process of phase transformation. Thus EE has been observed during changes of the state of aggregation or modification (first order phase changes, e.g., transition from the hexagonal to the cubic form in thallium, change or modification of hexagonal chromium and selenium, melting of ice, lead, tin, etc.) and during changes of orientation (second order phase changes, e.g., the antiferromagnetic-paramagnetic transition in $\mathrm{Ni}+\mathrm{NiO}$ and $\mathrm{Cr}$ $+\mathrm{Cr}_{2} \mathrm{O}_{3}$ ). The EE intensity is regarded as proportional to the defect content and a measure of the degree of completion of the phase change. The mechanism is believed to be the same as that for stress-induced EE. The emission has also been observed during 
second-order phase changes in crystalline pyroelectrics (e.g., lithium niobate, $\mathrm{BeO}$ ), where the mechanism is regarded as a thermally stimulated field effect (see Glaefeke 1979 for further details).

\subsection{Theoretical models for $E E$}

It is clear from the preceding description that $\mathrm{EE}$ is a rather complex phenomenon involving several intermediate steps, depending on the type of material and the actual experimental condition. However, there appear to be three broad steps in the electron emission mechanism: (i) External excitation generates defects and the relaxation of the response to the excitation is associated with the motion or relaxation of these defects. (ii) The defects transfer their energy either directly or via some other agent (e.g., surface plasmons in metals, and possibly phonons) to the electrons by the process of annihilation, or rearrangement, or by chemical reactions (at the surface). (iii) Electrons gaining this energy escape the material in the form of EE when their energy exceeds the work function or the electron affinity, or when an electric field (in electrified fissures) assists the emission. The controlling factors here (e.g. work function, etc.) may themselves depend on the nature of the excitation and on the experimental conditions.

Glaefeke (1979) has discussed various theoretical models for the last two steps, especially for dielectrics. Following Kelly (1972), he has described the thermionic emission model, in which the electron concentration is determined by reaction kinetics controlled by the concentration of defects (electron traps) and the retrappingrecombination processes for electrons. The theory has been applied to TSEE with some success. A field-assisted emission model has been developed for space-chargecontrolled EE from highly insulating materials (excitation induced by electron bombardment) with the transport and escape of electrons determined by a MonteCarlo process. The model can explain the intensity, energy and angular distribution of EE, for example in BeO (Kortov and Zolnikov 1975; Fitting et al 1979).

In EE from metals with or without an oxide layer, most of the theoretical work has been devoted to step one. Early studies on EE were on the diffusion of vacancies or point defects towards the surface. The rate of arrival of the defects at the surface was determined by Fick's law. This was then assumed to be proportional to the rate at which the electron emitting centres were activated and thereby to the electron emission rate (Grunberg and Wright 1955; von Voss and Brotzen 1959; Pimbley and Francis 1961; Claytor and Brotzen 1965). More recently Nagornykh and Demin (1978) have incorporated into the diffusion equation the generation of vacancies during the straining of oxide coated metals. The diffusion of point defects from the metal into the oxide was matched by the diffusion of oxygen in the opposite direction. The formation of a filled electron trap ( $F$-centre type) at the oxygen vacancy in the oxide was assumed. The electron emission rate was determined from the reaction kinetics involving thermal ionization of traps and recapture of electrons by the traps. The model was applied to EE from oxidised $\mathrm{Al}$ with some success.

The model for EE in bursts is based on crack propagation, and is more phenomenological than the model described above (Arnott and Ramsey 1971; Rosenblum et al 1977a). These however apply specifically to oxide coated metals. For surface-controlled EE, models based on chemisorption and Auger transitions have been evolved (Kasemo et al 1979). Models based on the stress dependence of thermionic emission have also been considered (Tinder 1968). 


\section{Acoustic emission}

By acoustic emission (AE) one means the generation of stress waves during structural changes in a material. These may be generated internally (eg., during phase transitions) or by an externally applied stress. The phenomenon is known to occur for almost all kinds of materials, and has been called by different names in different fields of research; for example, it is referred to as 'seismoacoustic activity' when observed in rocks under stress. AE usually refers to all kinds of stress waves even though the emitted frequencies may be far above the audible range. Extensive work has been done in this field, although to a somewhat applied nature (especially in relation to seismic studies of rocks), and some excellent review articles are available (Green 1969; Liptai et al 1971; Dunegan and Tatro 1971; Lord 1975). A brief description of the relevant parts of the subject follows:

The first systematic study of AE was by Kaiser in the 1950's, on polycrystalline zinc, steel, aluminium, copper and lead. He observed that AE was irreversible, i.e., emission did not occur during reloading of a material until the stress exceeded its previous value - a phenomenon usually known as the 'Kaiser effect'. This memory effect applies to most metals but generally not to other materials. In 1961 Schofield reported AE from aluminium and zinc single crystals, commercial copper, aluminium, lead and 70-30 brass, and established correlation with dislocations, slips and grain boundary motion. Tatro and Liptai (1962) studied AE from polycrystalline aluminium and steel for various strain values, and from the observed dependence on the surface condition of the specimen, concluded that AE was a surface phenomenon. Later Schofield studied AE from aluminium single crystals, with and without the oxide layer, and found the surface condition to be of secondary importance except for influencing the strain locally at the point where AE commenced in a burst. It was found that the oxide coating was not a source of AE. Further work by Schofield has established AE as primarily a volume effect. The general features of AE derived from the work of several persons can be summarized as follows (Lord 1975).

AE occurs in two forms: continuously, and in bursts. Continuous emissions of rather high frequency $\left(z 10^{2} \mathrm{kHz}\right)$ and very low intensity usually occur in metals and metallic systems, and supposedly arise from mobile dislocations and possibly slip movements. Burst emissions occur with much higher intensity and are supposedly associated with failures such as twinning, microcracks, unpinning of dislocations from obstacles, and growth of already existing cracks; they occur at relatively large values of the plastic strain.

Figure 7 shows a typical experimental AE rate as a function of the tensile strain (Dunegan and Harris 1969). This particular result is remarkable in that it fits beautifully with Gilman's mobile dislocation model (Gilman 1966) which gives the mobile dislocation density $N_{m}$ as a function of the plastic strain $\varepsilon_{p}$ through the relation $N_{m}=m \varepsilon_{p} \exp \left(-\phi \varepsilon_{p}\right)$, where $m$ is the dislocation breeding factor, and $\phi=H / \sigma$, where $H$ is the hardening coefficient, and $\sigma$ is the rms stress. The fit is shown in the dashed curve in figure 7 with $m=1.44 \times 10^{7}$ and $\phi=70$ (Lord 1975). This shows that the AE rate is directly related to the mobile dislocation density, so that one may contemplate determining $H$, an important mechanical parameter, using the AE technique. Unfortunately such a fit of Gilman's formula does not work for many materials. This has been attributed to the inhomogeneity in the strain caused by the occurrence of Lüder's bands.

At present there does not seem to be any rigorous microscopic theory for the actual 


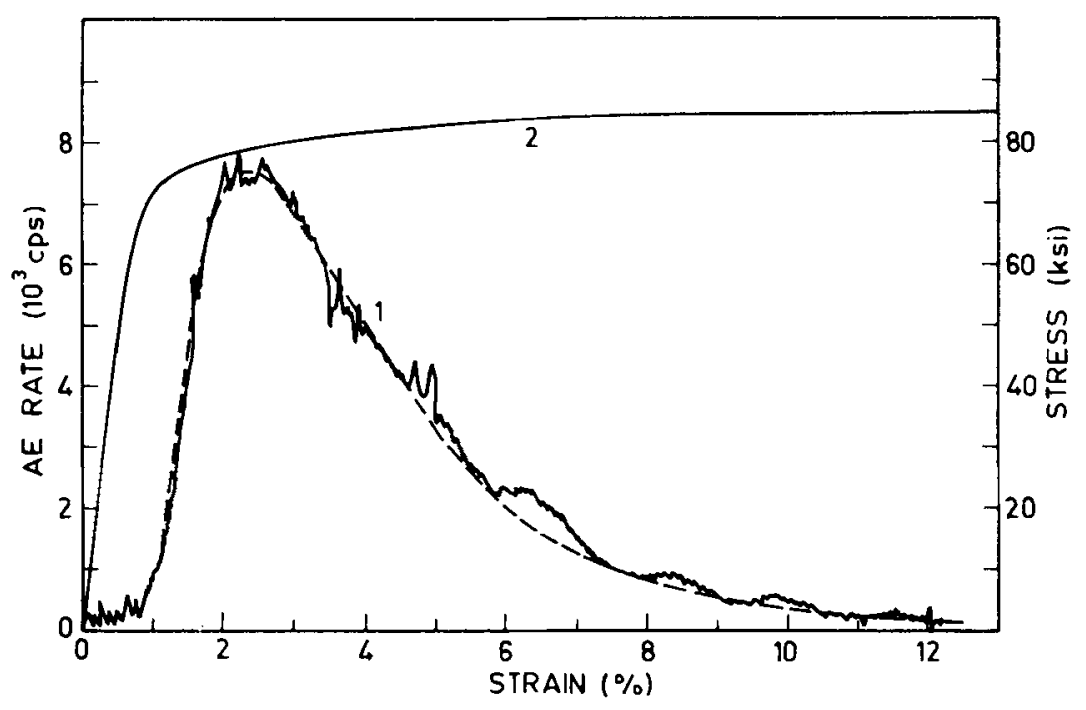

Figure 7. Acoustic emission intensity vs strain: 1. experimental: dashed curve. Gilman's relation. Stress vs strain: 2. (see Lord Jr 1975).

internal mechanism of AE. The major thrust in this area is in the direction of applied research, of establishing empirical relationships between AE and mechanical parameters (Lord 1975). Further, there has been no attempt to correlate AE with EE, although the typical emission intensity vs strain curves for $\mathrm{Al}$ are remarkably similar (compare figures 2 and 7).

\section{Mechanoluminescence}

Some materials emit light when subject to stress beyond a particular level. This phenomenon is broadly called mechanoluminescence $(\mathrm{ML})$ or triboluminescence. $(\mathrm{ML}$ is preferred, to avoid confusion with thermoluminescence which is customarily denoted by $\mathrm{TL}$ ). Extensive research has been done in this field on a very large number of materials, and the subject has been reviewed by a number of authors (Meyer et al 1970; Walton 1977; Zink 1978). Only a brief account of the relevant aspects is given here.

The most widely-studied materials are the alkali halides where the electron traps are the source of luminescence. ML spectra are similar to the TL spectra of a given material. Belyaev et al (1963) observed ML spectra during the growth of cracks in alkali halides under uniaxial strain, with photon emission in bursts, indicating that ML is associated with crack propagation. $M L$ during fracture can be a hundred times more intense than TL, the concept of electrified fissures has been used to explain this (Belyaev et al 1966; Belyaev and Martyshev 1969). Observations on ML during abrasion or scratching of alkali halides have been correlated with the propagation of cracks and TL (estimated temperatures as high as $10^{4} \mathrm{~K}$ ) at the crack-tip (Meyer et al 1970). ML emission has been found to have a memory (analogous to the Kaiser effect) in activated alkali halides and $\mathrm{ZnS}$. In experiments where the strain is repeated, no emission is observed in the elastic or plastic regions; and for a second application of strain, not until its magnitude exceeds that of the previously applied strain. This indicates that the $M L$ intensity depends on the 
depinning of dislocations under stress (Alzetta et al 1970; Scarmozzino 1971). Simultaneous ML and AE have been observed during mechanical deformation of organic glasses, steel and copper plates, indicating a common origin (Sodomka et al 1980). ML during the deformation and fracture of metallic alloys has also been reported (Borisova et al 1975). Molotskii (1978) observed ML during the plastic deformation and fracture of copper. Zink and his coworkers have observed $\mathrm{ML}$ in many organic and inorganic crystals (Zink 1978; Chandra and Zink 1980). In many cases, explanations on the basis of piezo-electrification, cleavage electrification and triboelectrification, have been given (Meyer et al 1970; Walton 1977; Chandra 1981).

Intense ML has been observed from coloured alkali halides (coloured by $\mathrm{x}$ - or $\gamma$-ray irradiation) by a number of workers. The emission is thought to be a result of the interaction of dislocations with colour centres (Butler 1966; Shmurak 1969; Alzetta et al 1970; Guerrero and Alvarez-Rivas 1978; Chandra and Zink 1980). Recently ML emission during the application as well as the release of uniaxial compression in $\mathrm{x}$-ray irradiated alkali halides have been reported by Chandra and Elyas (1978). They have also studied $\gamma$-irradiated alkali halides and ML emission from coloured alkali halides under repeated stress, and established a linear relationship between the $\mathrm{ML}$ intensity and the measured density of newly-created dislocations at the end of repeated-stress cycles (Elyas 1981). At present a proper theoretical understanding of ML emission, particularly from materials containing active luminescence centres, is still lacking, although some theoretical attempts have been made to understand $\mathrm{ML}$ in terms of electric field and pressure effects (Lin et al 1980). Studies correlating ML with EE or AE also seem to be inadequate.

\section{Conclusions}

We have tried to present an introduction to the subject of some special physical effects, especially exoelectron emission, in the context of the mechanical deformation of solids. For a better understanding of the exoelectron process, we have also discussed possible mechanisms for EE and two other related phenomena, AE and ML. While a number of possibly important references may have been inadvertently omitted, it is hoped that this article is sufficiently self-contained to generate interest in studying EE in a systematic manner. It can be safely said that, at present, a concrete theory of EE is lacking. Further, extensive, experimental study is required for understanding and correlating EE with $\mathrm{AE}$ and ML. For example, one should study more carefully the role of the work function, dislocations (the applicability of Gilman's theory or modification thereof), the mechanism of energy transfer to electrons, and the escape of electrons from metals and oxide-coated metals. On the applications side, one ought to study more extensively the growth of microcracks using spot scanners (PSEE, as in Baxter 1973) and establish empirical relationships with mechanical parameters in the same manner as has been done for acoustic emission studies.

Further references on EE are: Scharmann (1967), Becker (1972), Ramsey (1976), Krylova (1976) and Glaefeke (1979).

\section{Acknowledgements}

The author thanks Dr G Srinivasan and Prof. S Ramaseshan for bringing this subject to 
his attention. Thanks are also extended to Mr C V Krishna Murthy for providing some of the material presented here.

\title{
References
}

\author{
Alzetta G, Chudacek I and Scarmozzino R 1970 Phys. Status Solidi 21775 \\ Arnott D R and Ramsey J A 1971 Surf. Sci. 281 \\ Baxter W J 1973 J. Appl. Phys. 44608 \\ Baxter W J and Rouze S R 1978 J. Appl. Phys. 494233 \\ Becker K 1972 CRC Crit. Rev. Solid State Sci. 374 \\ Belyaev L M, Nabatov V V and Martyshev Yu N 1963 Sov. Phys. Crystallogr. 7464 \\ Belyaev L M and Martyshev Yu N 1969 Phys. Status Solidi 3457 \\ Belyaev L M, Nabatov V V, Pisarevskii Yu V and Shaldin Yu V 1966 Sov. Phys. Crystallogr. 10647 \\ Bichevin V, Käämbre H and Lushchik Ch. 1971 Phys. Status Solidi a5 525 \\ Bohun A 1961 Czech. J. Phys. 11819 \\ Bohun A, Sak J and Psenickova M 1965 Czech-Z-Phys. 15667 \\ Borisova E A, Glebova R D, Platonov A A and Skyarov N M 1975 Sov. Phys. Dokl. 20435 \\ Born D and Linke E 1976 Proc. 5th Int. Symp. on EE and Dosimetry (eds) A Bohun and A Scharmann (Prague: \\ Inst. Solid St. Phys. Acad. Sci.) p 265 \\ Brotzen F R 1967 Phys. Status Solidi 229 \\ Brunsmann U and Scharmann A 1977 Phys. Status Solidi a43 519 \\ Brus L E and Comas J 1971 J. Chem. Phys. 542771 \\ Buck O, Pardee W J, Szalkowski F J and Thompson D O 1977 Appl. Phys. 12301 \\ Butler C G 1966 Phys. Rev. 141750 \\ Chandra B P 1981 Phys. Status Solidi a64 395 \\ Chandra B P and Elyas M 1978 Kristall. U. Tech. 131341 \\ Chandra B P and Zink J I 1980 Phys. Rev. B21 816 \\ Chrysson E and Holzapfel G 1980 Phys. Status Solidi 259 K49 \\ Claytor R N and Brotzen F R 1965 J. Appl. Phys. 363549 \\ Delchar T A 1967 J. Appl. Phys. 382403 \\ Dickinson J T, Braunlich P, Larson L A and Marceau A 1978 Appl. Surf. Sci. 1515 \\ Dunegan H L and Harris D O 1969 Ultrasonics 7160 \\ Dunegan H L and Tatro C A 1971 Techniques of metals research (ed) R F Bunshah (New York: Wiley \\ Interscience) Vol V p 273 \\ Elyas M 1981 Studies of mechanoluminescence produced during application and release of a uniaxial pressure in \\ $x$-irradiated $\mathrm{KBr}, \mathrm{KCl}, \mathrm{Kl}, \mathrm{LiF}$ and $\mathrm{NaCl}$ crystals, Ph.D. Thesis, The Ravishanker University, Raipur, \\ India
}

Euler M and Scharmann A 1976 Phys. Status Solidi a34 297

Fitting H J 1981 Phys. Status Solidi a63 K47

Fitting H J, Glaefeke H, Wild W and Lange J 1977 Phys. Status Solidi $\mathbf{2 4 2}$ K 75

Fitting H J, Glaefeke H, Wild W, Franke M and Müller W 1979 Exp. Tech. Phys. 2713

Ford L H, Holzapfel G and Kaul W 1970 Z. Angew Phys. 30259

Gesell T F, Arakawa E T and Calcott T A 1970 Surf. Sci. 20174

Gieroszynski A and Sujak B 1965 Acta Phys. Pol. 28311

Giimann J J 1966 Proc. 5th U.S. Nat. Congr. Appl. Mech. 385

Glaefeke H 1979 Topics in applied physics (ed) P Bräunlich (Berlin: Springer-Verlag) Vol 37 p 224

Green A T 1969 Nucl. Safety 104

Grunberg L 1958 Br. J. Appl. Phys. 985

Grunberg L and Wright K H R 1955 Proc. Roy. Soc. London A232 403

Guerrero E and Alvarez-Rivas J L 1978 Solid State Commun. 28199

Hiernaut J P, Forier R P and Van Cakenberghe J 1972 Vacuum 22471

Holzapfel G and Krystek M 1976 J. Phys. (Paris) 37 C-7-238

Jakowski N and Glaefeke H 1976 Thin Solid Films 36195

Kasemo B, Tornqvist E, Nфrskov J K and Lundqvist B T 1979 Surf. Sci. 89554

Kelly P 1972 Phys. Rev. B5 749

Kelley P and Himmel L 1976 Comm. Solid State Phys. 781 
Kirihata H and Akutsu J 1979 Phys. Status Solidi a51 K 109

Komai K 1978 Trans. Jap. Inst. Met. 19119

Kortov V S and Myasnikov I E 1972 Phys. Status Solidi a9 119

Kortov V S and Zolnikov P P 1975 Phys. Status Solidi a31 331

Kortov V S, Mints R I, Myasnikov I E and Gaprindoshvily A Y 1970 Phys. Status Solidi a 13

Kortov V S, Mints R I and Teplov V G 1971 Phys. Status Solidi 27 K89

Kortov V, Isakov V, Glaefeke H and Fitting H J 1982 Phys. Status Solidi a73 K275

Kramer J 1950 Der metallische zustand (Gottingen: Vanderhoeck Ruprecht)

Kriegseis W and Scharmann A 1975 Phys. Status Solidi 229407

Krishna Murthy C V 1982 Exoelectron emission, M.Sc. Thesis, Indian Institute of Technology, Bombay, India

Krylova I V 1971 Phys. Status Solidi 27359

Krylova I V 1976 Usp. Biol. Khimii 452138 (English Transl.: 1976, Russ. Chem. Rev. 45 1101)

Krylova I V, Svitov V I and Konyushkina N I 1976 Zh. Fiz. Khimii 50933 (English Transl.: 1976 J. Phys. Chem. 50 555)

Lewis T J 1954 Proc. Phys. Soc. B67 187

Lin S H, Wutz D, Ho Z Z and Eyring H 1980 Proc. Natl Acad. Sci. (USA) 771245

Linke E $1970 Z$. Angew Phys. 29241

Liptai R G, Harris D O, Engle R B and Tatro C A 1971 Int. J. Nondestruct. Test. 3215

Lord A E Jr. 1975 Physical acoustics (eds) W P Mason and R N Thurston (Academic Press: New York) Vol. XI 289

McCarrol B 1969 J. Chem. Phys. 504758

McLennan J C, 1902 Philos. Mag. 3195.

Maiste A, Sorkin B, Elango M and Käämbre H 1973 Phys. Status Solidi a20 K83

Meyer K, Obrikat D and Rossberg M 1970 Kristall. Tech. 5 5, 181

Mints R I, Melekin V P, Segal V M and Icolev I Yu 1973 Sov. Phys. Solid State 142153

Molotskii M I 1978 Sov. Phys. Solid State 20956

Mueller R K and Pontinen K 1964 J. Appl. Phys. 351500

Nagornykh S N and Demin Yu A 1978 Phys. Met. Metall. 46170

Nink R and Holzapfel G 1973 J. Phys. (Paris) 34 C-9-491

Pardee W J and Buck O 1977 Appl. Phys. 14367

Pimbley W T and Francis E E 1961 J. Appl. Phys. 321729

Rabinowicz E 1977 Sci. Am. 23674

Ramsey J A 1976 Progr. surface and membrane Sci. (New York: Academic Press) Vol 11 p 117

Rhead G E 1977 Surf. Sci. 6820

Rosenblum B, Carrico J P, Bräunlich P and Himmel L 1977 J. Phys. E10 1056

Rosenblum B, Braunlich P and Himmel L 1977a J. Appl. Phys. 485262

Rudolf G and Glaefeke H 1982 Phys. Status Solidi a 73 K253

Scarmozzino R 1971 Solid State Commun. 91159

Scharmann A 1967 Festkorperprobleme (Berlin: Akad.-Verl.) Vol 6 p. 106

Scharmann A and Wiessler U 1980 Phys. Status Solidi 960117

Schlenk W 1976 Phys. Status Solidi a33 217

Shmurak S Z 1969 Sov. Phys. Solid State 101526

Shorshorov M Kh., Zhebynev D A and Alekhin V P 1976 Proc. Sth Inter. Symp. on EE and Dosimetry (ed) A Bohun and A Scharmann (Prague: Inst. Solid St. Phys. Acad. Sci.) p. 229

Sodomka L, Leistner M, Tessmar H and Karel F 1980 Kristall. U. Tech. 151101

Sujak B 1964 Acta Phys. Pol. 261033

Sujak B and Gieroszynski A 1970 Acta Phys. Pol. A37 733

Sujak B, Gieroszynski A and Pega E 1965 Acta Phys. Pol. 2861

Sujak B, Gieroszynska K and Gieroszynski A 1982 Phys. Status Solidi 272 K177

Tale I, Kortov V and Popov V 1982 Phys. Status Solidi a74 395

Tatro C A and Liptai R G 1962 Proc. Symp. on Physics and Non Destructive Testing (Southwest Research Inst., San Antonio, Texas) p. 145

Tinder R F 1968 J. Appl. Phys. 39355

Von Voss W D and Brotzen F R 1959 J. Appl. Phys. 301639

Walton A J 1977 Adv. Phys. 26887

Wortmann J 1978 Phys. Status Solidi a50 509

Zink J I 1978 Acc. Chem. Res. 11289 


\section{Discussion}

M Yussouff: Has the velocity distribution of the emitted electrons been measured?

G Mukhopadhyay: Yes, it has, in some cases.

R Chidambaram: How does thermoluminiscence dosimetry compare with exoemission?

Mukhopadhyay: I am sorry, I can't give you a precise answer.

R Krishnan: If there are two or more processes of exoemission occurring simultaneously, can one separate one from the other?

Mukhopadhyay: Only by doing additional experiments.

$G$ Venkataraman: Would the exoemission rate depend on the type of defect migrating to the surface?

Mukhopadhyay: Yes, it would-particularly the temperature dependence, because activation is involved.

C K Majumdar: How long before the actual crack occurs can one detect anything by the techniques you have described?

Mukhopadhyay: This depends on the material. In fatigue cycling with a large stress amplitude, very early detection is possible (at $0.7 \%$ of failure).

P Rodriguez: Is the exoemission phenomenon more amenable to measurement in fatigue cycling than in a uniaxial tensile test?

Mukhopadhyay: Not necessarily, but I think this depends on the experimentalist.

M Yussouff: Is exoemission a field emission process?

Mukhopadhyay: No, because the actual explanation depends on the type of sample and environment involved. 